\title{
Drug interactions with QT-prolonging antibiotics: an epidemiological study in community pharmacies
}

\author{
Eline Vandael ${ }^{1 *}$, Chantal Leirs ${ }^{2}$, Margaux Claes $^{3}$, Amelie Matheve $^{3}$, Elien Verbeeck ${ }^{3}$, Delphine Demeyer ${ }^{3}$, \\ Veerle Foulon ${ }^{4}$
}

From Methods in Epidemiology Symposium

Leuven, Belgium. 17 September 2015

\section{Background}

More than 170 drugs of different therapeutic classes are linked with a prolongation of the QTc-interval, which in rare cases can lead to Torsade de Pointes (TdP) and sudden cardiac death. The risk is especially high in patients with other risks factors for QTc-prolongation or when two or more QTc-prolonging drugs are combined.

\section{Aims}

To investigate the use of QT-prolonging antibiotics, concomitant risk factors for QT-prolongation and the current management of these interactions in community pharmacies.

\section{Methods}

An epidemiological study on data of a dispensing database of Flemish community pharmacies (Surplus network; $\mathrm{N}=100$ ) was performed. The patients who received a QT-prolonging antibiotic in the last week of May 2014 were selected and their medication histories (FebruaryMay 2014) were screened for other QT-prolonging drugs and concomitant risk factors (summarized in a risk score: $=65$ years, female, cardiovascular disease, diabetes, thyroid disturbances, each 1 point; potassium-lowering diuretics: 3 points; antiarrhythmic drugs: 4 point). Furthermore, the management of QT-signals by the pharmacist was analyzed.

\section{Results}

In the study period, 928 patients $(56.4 \%$ females, median age 55.5 years) received a QT-prolonging antibiotic (especially azithromycin, cipro/moxifloxacin). Of these patients, 313 (33.7\%) were synchronously treated with another QT-prolonging drug (of whom 67 patients with a drug with a known risk of TdP). Moreover, 107 of the 313 patients $(34.2 \%)$ had a risk score $=5$. A drug-drug interaction signal only turned up in 5 (of the 67) patients (because the warning system was turned off in most pharmacies) and the general practitioner was only contacted in one of these cases.

\section{Conclusion}

There is a high prevalence of interactions with QT-prolonging antibiotics in community pharmacies. However, the management and awareness of these interactions is limited. We are currently developing a decision support system to help pharmacists handling these interactions.

\section{Authors' details}

${ }^{1} \mathrm{KU}$ Leuven, Leuven, Belgium. ${ }^{2}$ Escapo NV, Mechelen, Belgium. ${ }^{3}$ 2nd Master Pharmaceutical Care, Faculty of Pharmaceutical Sciences, KU Leuven, Leuven, Belgium. ${ }^{4}$ Departement Pharmaceutical and Pharmacological Sciences, KU Leuven, Leuven, Belgium.

Published: 17 September 2015

doi:10.1186/2049-3258-73-S1-P42

Cite this article as: Vandael et al:: Drug interactions with QT-prolonging antibiotics: an epidemiological study in community pharmacies. Archives of Public Health 2015 73(Suppl 1):P42.

'KU Leuven, Leuven, Belgium

Full list of author information is available at the end of the article 